\title{
OPTIMISATION OF A WAVE ENERGY CONVERTER
}

\author{
John Ringwood ${ }^{1}$ Shane Butler \\ Dept. of Electronic Eng., NUI Maynooth, Co. Kildare, \\ Ireland
}

\begin{abstract}
This paper examines a simple model of an idealised point-absorber wave energy device. The objective of the research is to examine how the device can be optimised in order to extract maximum energy from incident waves. This applies to both the broad design parameters of the buoy and also to the synthesis of the damping function, in which the wave energy is converted. The issue of the adjustment of the phase of the velocity evolution with respect to the incident force (known as phase control) is dealt with in detail, with the intention of optimising the damping force over the wave cycle. The research ultimately attempts to parameterise the optimal damping force in terms of incident wave frequency and device parameters. Copyright (c) 2004 IFAC
\end{abstract}

Keywords: Wave energy, heaving buoy, latching, optimisation

\section{INTRODUCTION}

Many researchers and practitioners have consider point absorber devices over the last two decades, including Budal and Falnes (Budal and Falnes, 1975), Wright et al (Wright et al., 2003) and Dick (Dick, 2003). Much of the literature has focussed on the issue of device optimisation through both basic device design (shape, mass distribution and buoyancy) and also the power take-off system used to extract the wave energy. The latter is the principal focus of interest in the current study and a number of researchers have addressed this point, with a particularly extensive study by Falnes (Falnes, 2002). Falnes makes a number of important conclusions:

(1) Energy conversion is maximised if the device velocity is in phase with the excitation force, and

\footnotetext{
1 This author would like to acknowledge the fruitful discussions with Prof. Bill Leithead of the Hamilton Institute at NUI Maynooth
}

(2) The velocity amplitude, $|u|$ should equal $\frac{F_{e}}{2 R_{i}}$, where $F_{e}$ is the excitation force and $R_{i}$ is a device resistance.

Condition 2 above has a number of difficulties:

- It requires that the wave excitation force, $F_{e}$, be measured,

- $R_{i}$ turns out to be a non-causal function, and

- The power take-off (PTO) machinery must supply energy during part of the wave cycle in order to achieve the optimum $|u|$.

The need to supply energy may be considered strange, but this can be likened to a person on a swing, who uses body and leg motion to increase the amplitude of the swinging, by appropriate timing of the effort. Nevertheless, the need to supply energy requires a very complex PTO system and to the best of this author's knowledge, such a system has not yet been realised. However, Condition 1, representing a passive requirement, has received considerable attention and several researchers have addressed the problem. In particular, a method used to delay the velocity evolution, 
called latching, has been employed by a variety of researchers (Falnes and Lillebekken, 2003; Babarit et al., 2003; Korde, 2002; Wright et al., 2003; Greenhow and White, 1997).

Note that Conditions 1 and 2 above can be alternatively formulated in terms of complex conjugate (Nebel, 1992) (or reactive) control, which considers the complex impedance of the device.

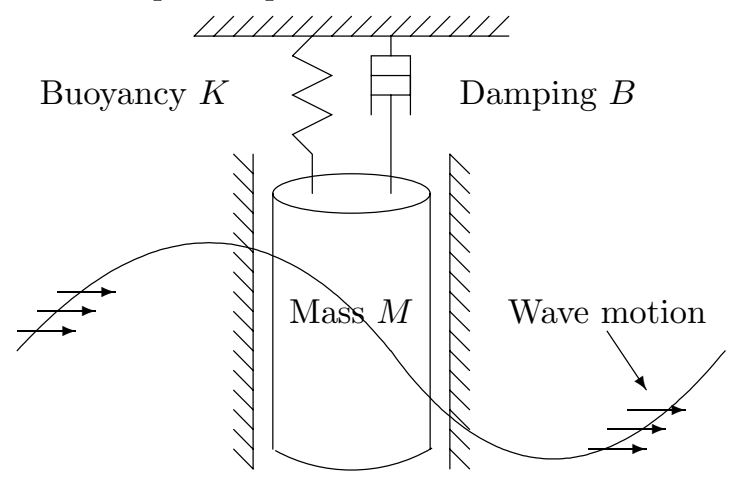

Fig. 1. Conceptual point absorber

A conceptual diagram of the device under examination is depicted in Fig.1. It consists of a cylindrical device which is constrained to move in the vertical direction (heave motion) only. The damping is notional and could be provided either internally in the device (as in (Korde, 2002)) or external to it (as in (Wright et al., 2003)). One other assumption in the concept device is that the diameter of the body is very small compared with the sea wavelength, so that the sea displacement is co-incident with the wave's heave force on the device.

\section{PRELIMINARY CALCULATIONS}

\subsection{System Model}

The dynamics of the devices described in Section 1 can be described, under some mild assumptions, by the differential equation:

$$
M \ddot{x}(t)+B \dot{x}(t)+K x(t)=F(t)
$$

where:

$x$ represents the displacement of the body from rest,

$M$ represents the mass (inertia) of the body,

$B$ represents the viscous friction, characteristic of hydraulic resistance used in PTO devices,

$K$ represents the buoyancy/gravity forces experienced by the body, and

$F(t)$ represents the heave force experienced by the wave-energy device.

There are a number of important assumptions inherent in the description in (1) above. In particular:
- Added mass, which affects both inertia and damping terms and is related to the inertia of the water surrounding the device, is largely ignored in the damping term in (1). However. it can be contained (notionally, at least) in $M$.

- Radiation damping, resulting from the energy carried away by surface waves generated by the device, is largely ignored. Some component of this can (notionally) be included as a linear term within $B$, though radiation damping is normally a nonlinear function of velocity. The linear damping component can represent skin friction, in particular (Fossen, 2002).

- The buoyancy/gravity restoring force is considered to be proportional to displacement from rest. In general, this is a small-signal approximation, since the restoring force is normally a nonlinear function of displacement.

The heave force due to incident waves will, in the first incidence, be assumed to be monochromatic, of the form:

$$
F(t)=A \sin \left(\omega_{w} t\right)
$$

The choice of monochromatic waves and the model in (1), is chosen for simplicity, since introduction of latching is a highly nonlinear intervention and quickly adds complexity. Equation (1) can also be easily recast in transfer function form as:

$$
\frac{X(s)}{F(s)}=G(s)=\frac{1}{M s^{2}+B s+K}
$$

or, in terms of transient response parameters, as:

$$
G(s)=\frac{1}{K} \frac{\omega_{n}^{2}}{s^{2}+2 \zeta \omega_{n} s+\omega_{n}^{2}}
$$

with

$$
\omega_{n}=\sqrt{\frac{K}{M}} \quad, \quad \zeta=\frac{B}{2} \sqrt{\frac{1}{M K}}
$$

Equation (1) can also be conveniently expressed in state-space (companion) form, as:

$$
A=\left[\begin{array}{cc}
0 & 1 \\
-\frac{K}{M} & -\frac{B}{M}
\end{array}\right] \quad B=\left[\begin{array}{c}
0 \\
\frac{1}{M}
\end{array}\right] \quad D=[0]
$$

with a state vector of:

$$
X(t)=\left[\begin{array}{c}
x(t) \\
\dot{x}(t)
\end{array}\right]
$$




\subsection{Power and Energy}

For a mechanical system, the power $(P)$ is the product of force and velocity. In wave energy systems, the PTO device is normally represented by the damper, giving the power developed in the damper as:

$$
P_{d}=\text { force } \mathrm{x} \text { velocity }=B \dot{x} \dot{x}=B \dot{x}^{2}
$$

The energy developed by the action on the damper over a period of time $t_{1}$ is:

$$
E_{d}\left(t_{1}\right)=\int_{0}^{t_{1}} P_{d} d t=\int_{0}^{t_{1}} B \dot{x}^{2} d t
$$

Maximum power is transferred to the damper when Equation (8) is maximised over a period of the wave force. This results in the condition:

$$
\omega_{n}=\sqrt{\frac{K}{M}}=\omega_{w}
$$

Under this maximum condition $\left(\omega_{n}=\omega_{w}\right)$, the velocity profile of the device is in phase with the wave force, consistent with Condition 1 in Section 1. Note that some adjustment of the device to achieve (9) may be possible through the use of appropriate quantity and position of water ballast.

The phase of the velocity profile (relative to the force profile) is evaluated as:

$$
\angle \frac{G(j \omega)}{s}=\frac{\pi}{2}-\tan ^{-1}\left(\frac{\omega B}{K-M \omega^{2}}\right)
$$

Clearly, if $K=M \omega^{2}$, then velocity is in phase with force or, indeed, if $B=\infty$. One further consideration here is that the force lags the velocity when:

$$
\omega_{w}<\omega_{n} \text { or } M \omega_{w}<K
$$

This places an upper bound on the device mass relative to the buoyancy. In addition, if a device is designed to be optimal for a given wave frequency, $\omega_{w}^{*}$, the wave force will only lag the velocity when the wave frequency, $\omega_{w}$ decreases below this value. This has important implications for the possibility of using latching to 'delay' the velocity profile in order to get it in phase with the force profile.

\section{LATCHING BASICS}

Latching can be achieved by means of a mechanical brake (applied at the appropriate latching points) or open close valves on the hydraulic lines of the PTO system. In simulation, latching can be achieved in two ways:
- The latching point is determined as the point where the device velocity goes to zero. At this point, the first and subsequent derivatives of displacement go to zero in (1) and if the wave force is replaced by a force equal and opposite to the restoring force $(K x)$, then 'latching' is achieved. This mechanism is illustrated in Fig.3.

- The closing of valve) can easily be implemented in simulation by setting the damping coefficient, $B$, to $\infty$.

Taking, for example, the case where $0.5 \omega_{n}=\omega_{w}$ (with $\omega_{w}=0.5$, corresponding to a wave period of just over 12 seconds), the response with latching (with $M=K=B=1$ ) is shown in Fig.3.

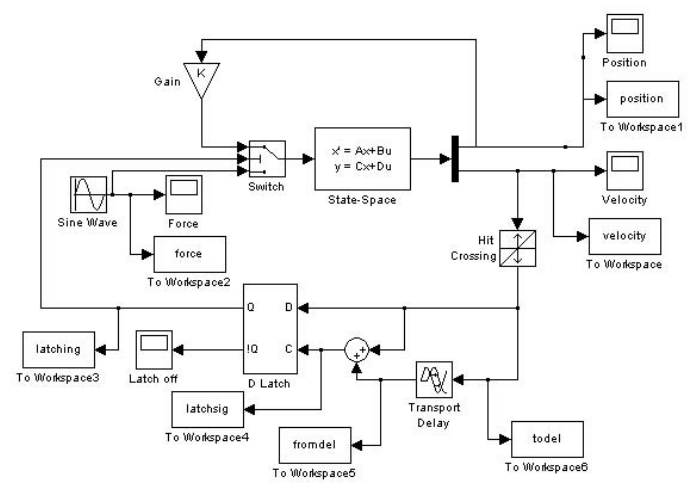

Fig. 2. Simulation configuration

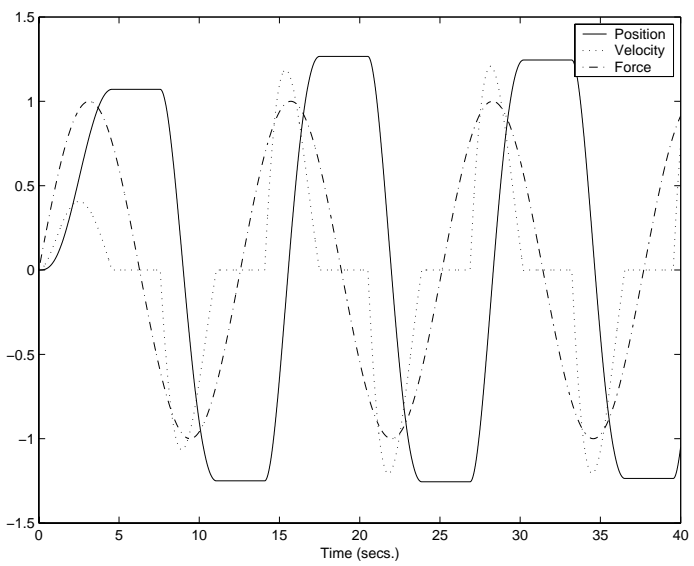

Fig. 3. Variations in $E_{d}$ and $T_{L}^{o p t}$ with $B$ and $\omega_{w}$

A number of features can be observed from Fig.3:

- The amplitude of the position response is greater then for the unlatched case,

- The velocity response, though highly nonlinear, is now in phase with the force profile, and

- The overall energy captured from the system, via the damper, has increased from $1.94 \mathrm{Ws}$ (unlatched) to 4.62 (latched) Ws per period of the incident wave.

Interestingly, the energy figure in the latching case is even greater than that achieved when $\omega_{n}=$ 
$\omega_{w}=1$ (at $3.14 \mathrm{Ws}$ ), but this is accounted for by the fact that the wave energy is proportional to wave period (Falnes, 2002) as:

$$
J=\frac{\rho g^{2}}{32 \pi} T H^{2}
$$

where $T$ is the wave period in seconds, $H$ is the wave height (trough to crest) in meters, and $\rho$ is the water density $\left(=1020 \mathrm{~kg} / \mathrm{m}^{3}\right.$ for sea water.

\section{SOLUTION TO LATCHING SYSTEM}

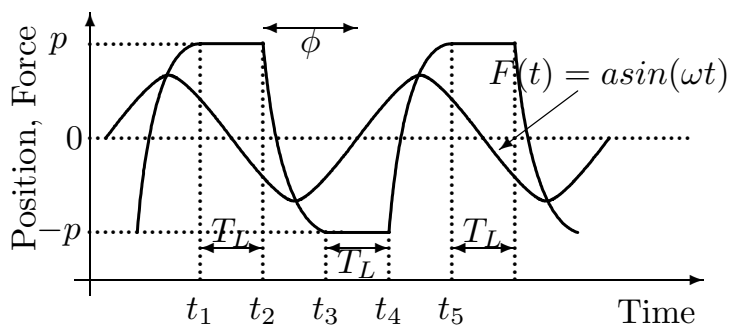

Fig. 4. Latching calculations

A solution to the latched system can be had by considering Fig.4. One period, or cycle, of the stimulus and response is given by:

$$
t_{5}-t_{1}=\frac{2 \pi}{\omega}
$$

Given that each of the latching periods occurs consistently for $T_{L}$ seconds, this gives the dynamic response period as:

$$
t_{3}-t_{2}=t_{5}-t_{4}=\frac{\pi}{\omega}-T_{L}
$$

For the (linear) system as given, the solution over the periods $t_{2} \rightarrow t_{3}$ and $t_{4} \rightarrow t_{5}$ is equal and opposite (assuming the transient response has died down). Therefore, the solution need only be evaluated over a half period. The solution for $t_{1} \rightarrow t_{2}$ is:

$$
X(t)=\left[\begin{array}{l}
p \\
0
\end{array}\right]
$$

The solution for the period $t_{2} \rightarrow t_{3}$ may be determined from the solution to (5), assuming a reference point of $t_{2}=0$, as:

$$
X(t)=e^{A t}\left[\begin{array}{l}
p \\
0
\end{array}\right]+\int_{0}^{t} e^{A(t-\tau)} B a \sin (\omega \tau+\phi) d \tau(16)
$$

Though equations (15) and (16) can be used to give an expression for the state (position and velocity) over the entire cycle, there are two unknowns: $\phi$, the phase offset between the force, $F(t)$, and

the position response, and

$p$, the height of the position response

However, since the response has zero mean, and the transient response has died down, we know that:

$$
X\left(t_{3}\right)=\left[\begin{array}{c}
-p \\
0
\end{array}\right]
$$

Inserting this in (16) gives:

$$
\begin{array}{r}
{\left[\begin{array}{c}
-p \\
0
\end{array}\right]=e^{A\left(\frac{\pi}{\omega}-T_{L}\right)}\left[\begin{array}{l}
p \\
0
\end{array}\right]+} \\
a \int_{0}^{\frac{\pi}{\omega}-T_{L}} e^{A\left(\frac{\pi}{\omega}-T_{L}-\tau\right)} B \sin (\omega \tau+\phi) d \tau
\end{array}
$$

Equation (18) represents 2 equations in 2 unknowns and can, in concept at least, be solved for $\phi$ and $p$. This type of solution procedure is followed in (Babarit et al., 2003), using a transfer function system description.

\section{LATCHING RESULTS}

Figs. 5 and 6 summarise the variations in the converted energy and optimal latching period (respectively) for variations in $B$ and $\omega_{w}$. Some comments are noteworthy:

- Converted energy decreases with increasing $\omega_{w}$ at smaller values of $B$, while it increases with $\omega_{w}$ at larger values of $B$.

- There is a clear optimal value for $B$, though this does seem to vary a little with $\omega_{w}$.

- At low $B$ values, the converted energy increases with $\omega_{w}$, as $\omega_{w}$ approaches $\omega_{n}$.

- The optimal latching period, $T_{L}^{o p t}$, goes to zero as $\omega_{w} \rightarrow \omega_{n}$ (in this case $\omega_{n}=1$.

- As stated above, there is little sensitivity of $T_{L}^{o p t}$ to variation in $B$, particularly for the range of $B$ shown.

- There is a clear $\frac{1}{\omega_{w}}$ relation with $T_{L}^{o p t}$ for all values of $B$. Re-plotting $T_{L}^{o p t}$ against $\frac{1}{\omega_{w}}$ for (as an example) $B=0.1$ shows a linear relationship between $T_{L}^{o p t}$ and the wave period (slope 0.5065, intercept -3.2022). As might be expected, $T_{L}^{o p t}$ does not appear as a consistent 'proportion' of the wave period, $T_{w}$, but rather is an affine function of $T_{w}$, with an offset of $2 \pi$ in the current example $\left(=\omega_{n}\right)$.

\section{THE OPTIMALITY OF LATCHING}

This paper focusses on latching as a solution to force the velocity profile to be in phase with 


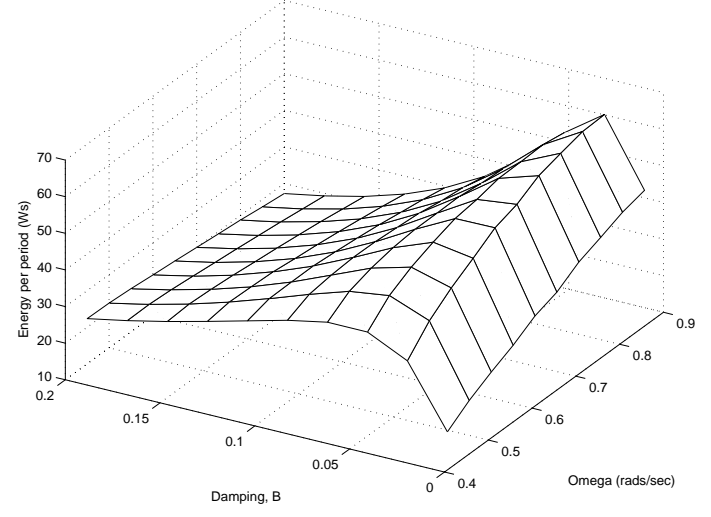

Fig. 5. Variations in $E_{d}$ with $B$ and $\omega_{w}$

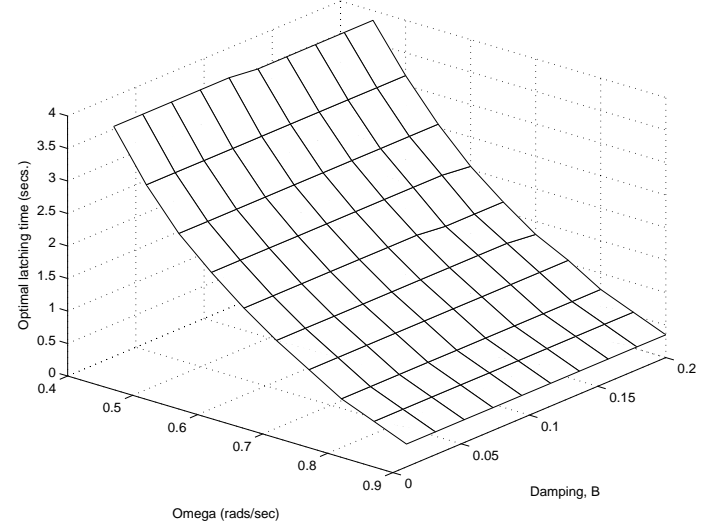

Fig. 6. Variations in $T_{L}^{o p t}$ with $B$ and $\omega_{w}$

the applied wave force. However, since the wave energy is converted in the damping term (see equation (8)) one can concieve of a multitude of loading possibilities, where the damping term is varied over the wave cycle, or scheduled with device velocity. Indeed, some researchers have looked at the possibility of having a very low damping 'load' at the beginning of the cycle (beginning at a point of zero velocity) and increasing the damping only after a preset velocity is reached. Such a 'freewheeling' strategy is in strong contrast to latching, where the damping is effectively infinite for the latching period i.e. a short period following the point of zero velocity.

To determine, in a limited way, the optimal loading regime, the damping term was parameterised in terms of a general sigmoid function (see Fig.7) as follows:

$$
B(t)=\frac{B_{\max }-B_{\min }}{1+e^{-\beta(t-t *)}}+B_{\min }
$$

This provides for many possible damping functions, including latching, freewheeling and uniform (linear) damping, as illustrated in Fig.8.

The parameters of the sigmoid in equation(18) were adapted, using a genetic algorithm (Goldberg, 1989), in order to maximise the energy function (equation (8), Fig.??) over a wave period, where $\omega_{w}=0.5$ (corresponding to a wave period of

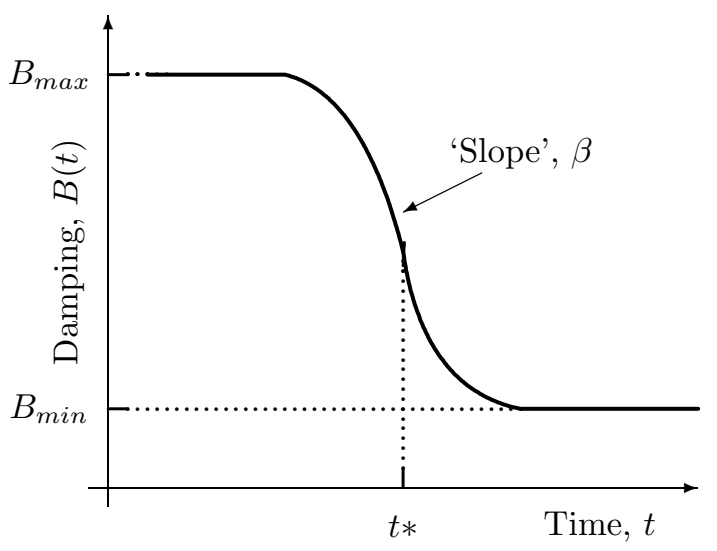

Fig. 7. Sigmoidal parameterisation of damping functions

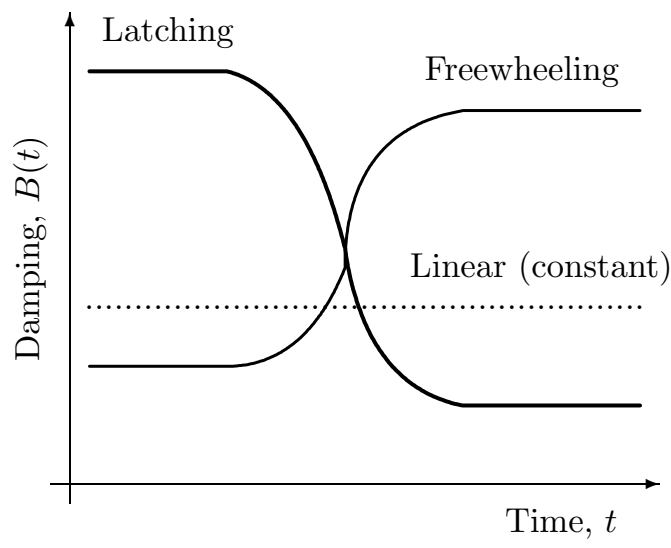

Fig. 8. Possible damping functions

\begin{tabular}{|c|c|}
\hline Parameter & Value \\
\hline Chromosome coding & Binary \\
Population size & 70 \\
Number of generations & 30 \\
Generation gap & 0.7 \\
Recombination probability & 0.7 \\
Mutation probability (per bit) & 0.035 \\
Selection & Roulette wheel \\
\hline
\end{tabular}

Table 1. GA parameters

\begin{tabular}{|c|c|c|c|c|}
\hline Range & $B_{\min }$ & $B_{\max }$ & $t *$ & $\beta$ \\
\hline Max & 0 & 0 & -20 & 0 \\
Min & 50 & $10^{90}$ & +20 & 3000 \\
\hline
\end{tabular}

Table 2. Range for sigmoid parameters

12.56 secs.). The default values of $M=K=1$ were used, as before. A genetic algorithm (GA), with elitism, was employed since the performance surface to be searched is non-convex with respect to the sigmoid parameters. Briefly, the parameters of the GA are given in Table 1.

The ranges allowed for the sigmoid parameters are given in Table 2. 


\begin{tabular}{|c|c|c|c|c|}
\hline Parameter & $B_{\min }$ & $B_{\max }$ & $t *$ & $\beta$ \\
\hline Final value & 0.0546 & $10^{90}$ & 2.7 & -2530 \\
\hline
\end{tabular}

Table 3. Final sigmoid parameter values

The final values attained following maximisation of the energy per wave period are as given in Table 3 .

These values clearly indicate the optimality of a latching strategy. Firstly, the value for $\beta$ is negative, indicating that a 'high then low' strategy (characteristic of latching) is preferable for $B(t)$. The height of the initial damping is at the upper limit of the allowed range, indicating effectively infinite initial damping (or complete 'latching'). The magnitude of the slope parameter, $\beta$ is very large, indicating an almost instantaneous transition from latching to a subsequent finite value for $B$. The final value for $B, B_{m i n}$, is very close to the optimal damping value indicated in Fig.5, for a wave frequency of $\omega_{w}=0.5$. Finally, the time for which $B(t)$ is held high (the latching time) is very close to the optimal value (for $\omega_{w}=0.5$ ) indicated in Fig.6.

\section{CONCLUSIONS}

Optimal extraction of wave energy requires a number of device aspects to be considered. In the first instance, it is required that the resonant frequency of the device be placed in the region of dominant wave frequency. Further to this, adjustments to optimise the power absorbed by the device in the damping element can be made by attempting to get the velocity profile in phase with the incident wave force. Latching provides one mechanism of achieving this and it can be shown that latching provides the optimum adjustment of the device damping over the wave period. Given that latching is employed, some further care needs to be taken in setting the appropriate (unlatched) damping level, since the energy take depends on this damping value, with the optimal damping also dependent on the dominant wave frequency. While the analysis in this paper has assumed a simplified device model, it focusses on the salient issues in wave energy device design and should be extendible to more realistic sea/device models for heaving buoys and could also be extended to more complicated structures, such as the McCabe Wave Pump (MWP) (McCormick et al., 1998).

\section{REFERENCES}

Babarit, A., G. Duclos and A.H. Clement (2003). Comparison of latching control strategies for a heaving wave energy device in a random sea. In: Proc. $5^{\text {th }}$ European Wave Energy Conference. Cork.
Budal, K. and J. Falnes (1975). A resonant point absorber of ocean wave power. Nature 256, 478-479.

Dick, W. (2003). Report on eu eureka project e! 2278 wwec. http://www.eureka.be/ifs/files/ifs/jsp-bin/ eureka/ifs/jsps/publicShowcase.jsp?fileTo Include=ProjectProfile.jsp\&docid=1483664.

Falnes, J. (2002). Ocean Waves and Oscillating Systems. Cambridge University Press. Cambridge, UK.

Falnes, J. and P.M. Lillebekken (2003). Budal's latching-controlled-buoy type wavepower plant. In: Proc. $5^{\text {th }}$ European Wave Energy Conference. Cork.

Fossen, T.I. (2002). Marine Control Systems: Guidance, Navigation and Control of Ships, Rigs and Underwater Vehicles. Marine Cybernetics. Norway.

Goldberg, D.E. (1989). Genetic Algorithms in Search, Optimisation and Machine Learning. Addison-Wesley.

Greenhow, M. and S.P. White (1997). Optimal heave motion of some axisymmetric wave energy devices in sinusoidal waves. Applied Ocean Research 17, 141-159.

Korde, U.A. (2002). Latching control of deep water wave energy devices using an active reference. Ocean Engineering 29(11), 13431355 .

McCormick, M.E., J. Murtagh and P. McCabe (1998). Large-scale experimental study of a hinged-barge wave energy conversion system. In: Proc. 3rd European Wave Energy Conference. Patras, Greece.

Nebel, P. (1992). Maximising the efficiency of wave-energy plants using complex-conjugate control. Journal of Systems and Control Engineering 206, 225-236.

Wright, A., W.C. Beattie, A. Thompson, S.A. Mavrakos, G. Lemonis, K. Nielsen, B. Holmes and A. Stasinopoulos (2003). Performance considerations in a power take-off unit based on a non-linear load. In: Proc. $5^{\text {th }}$ European Wave Energy Conference. Cork. 